\title{
On Renormalization and Complex Space-Time Dimensions
}

\author{
J. F. Ashmore \\ International Atomic Energy Agency \\ and \\ United Nations Educational Scientific and Cultural Organization \\ International Centre for Theoretical Physics, Trieste, Italy
}

Received July 31,1972

\begin{abstract}
The method of using the dimension of space-time as a complex parameter introduced recently to regularize Feynman amplitudes is extended to an arbitrary Feynman graph. The method has promise of being particularly well-suited to gauge theories. It is shown how the renormalized amplitude, together with the Lagrangian counter-terms, may be extracted directly, following the method of analytic renormalization.
\end{abstract}

\section{Introduction}

Of late, a number of authors suggested, independently, an approach to the renormalization of the perturbation expansion in Lagrangian quantum field theory which uses the dimension of space-time as a complex parameter [1-3]. The most important feature of the method is that the regularization procedure in general preserves the formal structure necessary for the theory to satisfy Ward-Takahashi identities appropriate to the gauge symmetries present. With exceptions, the argument relies on the observation that the Ward-Takahashi identities are formally independent of the space-time dimension ${ }^{1}$. The method of extracting renormalized results is very close in spirit to the method of analytic renormalization of Speer $[4,5]$, which, per se, does not preserve gauge symmetries [6].

Now the attractive feature of analytic renormalization is that the renormalized amplitude is defined non-recursively, but it is equivalent to the additive, recursive definition of Bogolubov, Parasiuk, and Hepp [7] (which is the most general treatment in Lagrangian quantum field theory). Both approaches have been shown to yield the Lagrangian counter-terms directly.

In Refs. [1-3], the method of regularization was only demonstrated by example in lowest orders. It is our purpose here to give a general

1 The exceptions are, for example, when the theory contains an axial coupling; it is then implicitly necessary that there be an odd number of space dimensions.

13 Commun. math. Phys, Vol. 29 
construction valid for any Feynman amplitude, such that the renormalized value may be extracted directly, as in analytic renormalization. This involves formally introducing different space-time dimensions for a certain set of subgraphs of the original Feynman graph, and then continuing the result in these dimensions. We feel this procedure is a complementary regularization technique to that in Refs. [4,5].

Our approach is to be compared with the philosophy of making subtractions recursively, as advocated in Ref. [3], where it becomes necessary to prove that the result is finite. The non-recursive definition to be given is obviously finite. Incidentally, the approach we adopt here is closely related to renormalizing an amplitude in Feynman parameter space [8]. In fact, the construction of the amplitude is made in such a representation, since there the dimensionality of the problem appears explicitly and is not dependent on graph-topological structure.

Section II is devoted to the construction of the regularized amplitude, and we state two theorems on the analytic structure of the amplitude as a function of these many "complex dimensions".

Section III gives the recipe for extracting the renormalized result; we state that this renormalization corresponds to an additive renormalization in the sense of Bogolubov-Parasiuk-Happ [7]. The construction of the appropriate counter-terms is relegated to Appendix B, while Appendix A contains a resumé of the necessary terminology. Throughout we shall be content with the mere statement of the theorems; it transpires that the renormalization problem is virtually identical with that of analytic renormalization in the sense of Speer. Proofs are fairly trivial modifications to the combinatoric machinery and methods developed extensively in Refs. $[4,5,7]$.

A complete formal proof that the renormalized theory satisfies Ward-Takahashi identities is beyond the scope of any approach to renormalization based on individual terms in the perturbation expansion.

\section{Construction of the Regularized Amplitude}

Let $G\left(V_{1} \ldots V_{m} ; \mathscr{L}\right)$ be a connected Feynman graph. The amplitude associated with $G$ is formally

$$
\mathscr{T}_{G}=\prod_{l \in \mathscr{L}} \Delta_{l}\left(x_{l_{i}}-x_{l_{f}}\right) .
$$

In $p$-space, this has the form (up to irrelevant constants)

$$
\tilde{\mathscr{T}}_{G}=\delta\left(\sum_{i=1}^{m} p_{i}\right) \int \prod_{i=1}^{N(G)} d^{4} k_{i} \prod_{l=1}^{L} Z_{l}\left(q_{l}\right)\left(q_{l}^{2}-m_{l}^{2}+i 0\right)^{-1}
$$


where $q_{l}$ is a linear combination of $k$ and external momenta $p$

$$
q_{l}=\sum_{i=1}^{N(G)} a_{l i} k_{i}+\sum_{i=1}^{m} b_{l i} p_{i}
$$

Here $L=\#(\mathscr{L}(G))$, and $N(G)$ is the number of independent circuits ("loops") of $G$.

In general $\left(\Pi Z_{l}\right)$ is an ordered polynomial (for example, with Dirac $\gamma$ matrices).

The Feynman parametric form of (2.2) is given by the following manipulations. We use the representation

$$
\begin{gathered}
i\left(q^{2}-m^{2}+i 0\right)=\int_{0}^{\infty} d \alpha \exp i \alpha\left(q^{2}-m^{2}+i 0\right) . \\
Z(q)=\left.\exp \left(q \cdot \frac{\partial}{\partial s}\right) Z(s)\right|_{s=0}
\end{gathered}
$$

allowing the "loop" integral ( $\Pi d k$ ) to be done in gaussian form by repeated use of the formula

$$
\int_{-\infty}^{+\infty} d p \exp i a p^{2}=\left(\frac{\pi}{a}\right)^{\frac{1}{2}} \exp \left(i \frac{\pi}{4} \frac{a}{|a|}\right) .
$$

The details are standard and one arrives at the result [9]

$$
\tilde{\mathscr{T}}_{G}=\operatorname{const} \delta(\Sigma p) \int_{0}^{\infty} \prod_{i=1}^{L} d \alpha_{i} C(\alpha)^{-2} \cdot \exp \left(i \frac{D(\alpha, p)}{C(\alpha)}\right) \cdot R(\alpha, p)
$$

where $C=\operatorname{det} \theta_{i j} ; D=\operatorname{det}\left(\begin{array}{cc}\theta_{i j} & \phi \\ \phi & \psi\end{array}\right)$ and we write

$$
\begin{aligned}
\theta_{i j} & =\sum_{l=1}^{L} \alpha_{l} a_{l i} a_{l j} \quad(i, j=1, \ldots, N), \\
\phi_{i} & =\sum_{l=1}^{L} \sum_{j=1}^{m} \alpha_{l} a_{l i} b_{l j} p_{j} \quad(i=1, \ldots, N), \\
\psi & =\sum_{l=1}^{L} \sum_{j, k=1}^{m} \alpha_{l} b_{l j} b_{l k} p_{j} \cdot p_{k}-\sum_{l=1}^{L} \alpha_{l}\left(m_{l}^{2}-i 0\right) .
\end{aligned}
$$

The spin factor $R$ is more complicated and given by [10]

$$
R(\alpha, p)=\left.\exp \left(\frac{i}{4} \sum_{i, j=1}^{N(G)}\left(\theta^{-1}\right)_{i j} \frac{\partial}{\partial s_{i}} \cdot \frac{\partial}{\partial s_{j}}\right) \prod_{l} Z_{l}\left(\sum_{l=1}^{N(G)} a_{l i} s_{i}+\chi_{l}\right)\right|_{s=0}
$$

with

$$
\chi_{l}=\sum_{j=1}^{m} b_{l j} p_{j}-\sum_{i=1}^{N(G)} a_{l i} \phi_{i}
$$


for $l=1,2, \ldots, L$. Here $p$ and $s$ are four-vectors with scalar product appropriate to Minkowski space $M_{4}$.

It may be shown that the integral (2.5) is independent of the choice of momenta $k$ [9]. It depends only upon the topology of the graph $G$.

The original divergences of (2.2) are reflected in divergences of the representation (2.5) at the lower limit of the $\alpha$ integrations, as $C(\alpha)$ vanishes with certain subsets of $\alpha$. We write $\tilde{\mathscr{T}}_{G}^{r}$ for the amplitude (2.5) with the replacement

$$
\int_{0}^{\infty} d \alpha_{i} \rightarrow \int_{r}^{\infty} d \alpha_{i} \quad(i=1, \ldots, L ; r>0) .
$$

We are going to construct the analogue of (2.5) when a certain set of subgraphs of $G$ carry additional independent components of momenta. How this works in the one- and two-loop case is demonstrated in Refs. [1-3]. These components are initially taken space-like, and the result continued in the number of dimensions which appear explicitly in the Feynman parametric form.

The set of graphs is the following:

Definition. Let $\mathscr{K}(G)$ be the set of dominant irreducible subgraphs $\hat{G}(U)$ of $G$ as $U$ runs over all generalized vertices of $G$ (cf. definitions A.2 and A.4). In general $G$ itself is contained in $\mathscr{K}(G)$. We call $\mathscr{K}(G)$ a divergence family, (d-family) for $G$. (The name simply reflects that every vertex part in additive renormalization [7] is associated to an element of $\mathscr{K}(G)$.)

Note that each $H \in \mathscr{K}(G)$ contains at least one circuit (i.e. $N(H) \geqq 1$ ).

To each $H \in \mathscr{K}(G)$ associate a non-negative integer $\omega_{H}$. The scalar product of two vectors $x, y$ in $\mathbb{R}^{H} \equiv \mathbb{R}^{\omega_{H}}$ will be denoted by $-(x, y)_{H}$. We shall construct the amplitude in $M_{\omega}=M_{4} \bigoplus_{H \in \mathscr{K}} \mathbb{R}^{H}$, i.e. the Minkowski space with $3+\sum_{H \in \mathscr{K}} \omega_{H}$ space-like dimensions. External four-vectors $\left(p_{\mu}\right)$ will be embedded in $M_{\omega}$ in the canonical way as $\left(p_{\mu}, \mathbf{0}\right)$.

By choosing a set of circuits $C_{1}^{H} \ldots C_{N(H)}^{H}$ for each $H \in \mathscr{K}(G)$, we may choose $\omega_{H}$ dimensional momenta $k^{H}$ in the subspace $\mathbb{R}^{H} \subset M_{\omega}$ associated with each such graph, so that the "line" momenta $q_{l}$ occurring in (2.1) become vectors in $M_{\omega}$; thus

$$
q_{l}=\left(\sum_{i=1}^{N(G)} a_{l i} k_{i}+\sum_{i=1}^{m} b_{l i} p_{i}\right)+\sum_{H \in \mathscr{K}} \sum_{i=1}^{N(H)} a_{l i}^{H} k_{i}^{H} .
$$

The $L(G) \times N(H)$ matrix $\left(a_{l i}^{H}\right)$ is defined by

$$
\begin{aligned}
a_{l i}^{H} & = \pm 1 \quad \text { if } \quad l \in C_{i}^{H} \\
& =0 \quad \text { otherwise }
\end{aligned}
$$


the sign depending upon the orientation chosen for the circuits. We define the matrix $\theta^{H}$ by

$$
\theta_{i j}^{H}=\sum_{l=1}^{L} \alpha_{l} a_{l i} a_{l j} \quad(i, j=1, \ldots, N(H))
$$

and set $C_{H}=C_{H}(\alpha)=\operatorname{det} \theta^{H}$.

Finally we change the differential in (2.2) to

$$
\left(\prod_{i=1}^{N(G)} d^{4} k_{i}\right) \rightarrow\left(\prod_{i=1}^{N(G)} d^{4} k_{i}\right)\left(\prod_{H \in \mathscr{K}} \prod_{i=1}^{N(H)} d^{\omega_{H}} k_{i}^{H}\right) .
$$

With the replacements (2.7) and (2.9), gaussian integration can again be done, with the result

where

$$
\begin{aligned}
\tilde{\mathscr{T}}_{G}^{r, \omega}= & \operatorname{const} \delta(\Sigma p) \int_{r}^{\infty} \prod_{i=1}^{L} d \alpha_{i} C(\alpha)^{-2} \exp \left(i \frac{D(\alpha, p)}{C(\alpha)}\right) \\
& \cdot\left(\prod_{H \in \mathscr{K}} C_{H}(\alpha)^{-\frac{\omega_{H}}{2}}\right) R(\alpha, p, \omega)
\end{aligned}
$$

$$
\begin{aligned}
R(\alpha, p, \omega)= & \exp \frac{i}{4} \sum_{i, j}\left[\left(\theta^{-1}\right)_{i j} \frac{\partial}{\partial s_{i}} \cdot \frac{\partial}{\partial s_{j}}+\sum_{H \in \mathscr{K}}\left(\left(\theta^{H}\right)^{-1}\right)_{i j}\left(\frac{\partial}{\partial s_{i}^{H}} \cdot \frac{\partial}{\partial s_{j}^{H}}\right)_{H}\right] \\
& \left.\cdot \prod_{l=1}^{L} Z_{l}\left(\sum_{j=1}^{H(G)} a_{l j} s_{j}+\sum_{H \in \mathscr{K}} \sum_{j=1}^{N(H)} a_{l j}^{H} s_{j}^{H}+\chi_{l}\right)\right|_{s=0}
\end{aligned}
$$

and $\omega$ is the set $\left\{\omega_{H}\right)_{H \in \mathscr{W}}$ of non-negative integers.

The integral in (2.10) has a complicated structure. We make the following crucial points:

a) All operations in $\Pi Z_{l}$ leading to scalar products of momenta are to be performed before the operations implicit in (2.11) (e.g. all traces should be evaluated). Scalar products are then reinterpreted as the natural scalar product in $M_{\omega}$.

b) All external momenta and indices are to be kept fixed appropriate to the canonical Minkowski space $M_{4} \subset M_{\omega}$, as above. This is implicit in $(2.10)$.

c) $R(\alpha, p, \omega)$ is implicitly a function of $\omega$ through the action of the differential operator in (2.11) subject to the remark a) above. It is at most a polynomial in $\omega$.

d) Although we have chosen a particular set of momenta $k, \tilde{\mathscr{T}}_{G}^{r, \omega}$ is in fact independent of this choice, depending only upon the topology of $G$.

e) In the case $\boldsymbol{\omega}=\mathbf{0}$ we formally recover the integral (2.5).

The essential importance of remarks a)-c) was demonstrated in Refs. [1-3]. We claim (2.10) is a natural generalization of the construction given there. 
We may now continue $\tilde{\mathscr{T}}_{G}^{r, \omega}$ in $\omega$ from the non-negative integers to $\mathbb{C}^{K}(K=\#(\mathscr{K}(G)))$. The (unique) continuation is given by (2.10). This is our definition of the regularized amplitude.

We now state some properties of $\tilde{\mathscr{T}}_{G}^{r, \omega}$. We omit the proofs which are but trivial modifications of those already given by Speer $[4,5]$.

Theorem 2.1. $\tilde{\mathscr{T}}_{G}(\omega)=\lim _{r \downarrow 0} \tilde{\mathscr{T}}_{G}^{r, \omega}$ exists for $\operatorname{Re} \omega_{H}$ sufficiently large and and negative, and defines by analytic continuation a function meromorphic in $\mathbb{C}^{K}$.

The analytic structure of $\mathscr{T}_{G}(\omega)$ may be made more precise by the following.

Theorem 2.2. $\mathscr{T}_{G}(\omega)$ admits the decomposition

$$
\mathscr{T}_{G}(\omega)=\sum_{\mathscr{E}} \mathscr{T}_{G}(\omega, \mathscr{E})
$$

the sum running over all s-families $\mathscr{E}$ of $G$ (cf. Definition A.3); $\mathscr{T}_{G}(\omega, \mathscr{E})$ has the property that

$$
\prod_{H \in \mathscr{E}}\{\Gamma(v(H))\}^{-1} \mathscr{T}_{G}(\omega, \mathscr{E})
$$

has an analytic extension to all of $\mathbb{C}^{K}$, where

$$
v(H)=-\frac{1}{2} N(H) \sum_{H^{\prime} \in \mathscr{K}(\hat{H})} \omega_{H^{\prime}}-\left[\frac{\mu(H)}{2}\right] .
$$

$(\mathscr{K}(\hat{H})$ is the d-family of the dominant graph $\hat{H}$ for $H$ in $G: c f$. Definition A.4.)

Note that Theorem 2.2 contains the conventional power-counting theorem as a special case [9].

\section{Renormalization}

In Section II we have shown how to construct an amplitude $\mathscr{T}_{G}^{\omega}$ which depends meromorphically on a set of parameters $\omega$ labelled by the $d$-family $\mathscr{K}(G)$ of $G$. The essential observation is that the analytic structure is virtually the same as in the analytic regularization of Speer $[4,5]$ where there the role of $\mathscr{K}(G)$ as an index set is played by the set of lines $\mathscr{L}(G)$ of $G$. This allows us to lift bodily all the combinatoric apparatus developed in Ref. [6].

The extraction of the finite part of the amplitude, together with the counter-terms can be achieved by the use of an analytic evaluator, which is a mapping of functions of the type described by Theorem 2.2 into functions analytic around a given point $\omega=\omega_{0}(=0$ here). 
An example of such an evaluator $\mathscr{V}$ is given by

$$
(\mathscr{V} f)(\boldsymbol{\omega})=\frac{(2 \pi i)^{K}}{K !} \sum_{s} \int_{C_{s(1)}} d \mu_{1} \ldots \int_{C_{s(K)}} d \mu_{k} \frac{f(\boldsymbol{\mu})}{\left(\mu_{1}-\omega_{1}\right) \ldots\left(\mu_{K}-\omega_{K}\right)}
$$

where if $0<R_{1}<\cdots<R_{K}<\mathscr{E}$ satisfy $\sum_{i<j} R_{i}<R_{j}, C_{i}$ is the contour $|z-1|=R_{i}$ oriented counter-clockwise and $\left|\omega_{i}\right|<R_{1}$. The sum runs over all permutations $s$ of $\{1,2, \ldots, K\}$.

The freedom to choose an analytic evaluator (but which must satisfy certain general requirements [6]) reflects itself in a finite change of the renormalizations.

Thus, let $\mathscr{T}_{G}(\omega)$ be constructed as in Section II and let $\mathscr{V}$ be an analytic evaluator. The analytically renormalized amplitude is given by

$$
\mathscr{T}_{G}^{\prime}=\lim _{\omega \rightarrow \mathbf{0}}\left(\mathscr{V} \mathscr{T}_{G}\right)(\omega) .
$$

The result is obviously finite.

Our main result is

Theorem 3.1. $\mathscr{T}_{G}^{\prime}$ is an additively renormalized amplitude.

The construction of the counter-terms is to be found in Appendix B. The proof that these counter-terms (vertex parts) are indeed a set for the additive renormalization of $\mathscr{T}_{G}$, in the sense of Definition A.7, is closely modelled on that of Ref. [6].

As stated in the introduction, a complete proof that we are ultimately led to gauge-invariant results is beyond the scope of our approach here. However, the regularized integral of Section II was constructed so that each graph in the $d$-family $\mathscr{K}(G)$ separately preserves structure. Now by explicit calculation it has been demonstrated [1-3] that the counterterm for the one-circuit graph is consistent with gauge-invariance requirements. This, coupled with the fact that the vertex parts of Appendix B satisfy a recursive structure, leads to the plausible conclusion that the necessary structure is preserved. Of course, the same evaluator should be used throughout the renormalization of the theory.

Acknowledgments. I am indebted to the participants of the Informal Conference on Renormalization held at Marseille, June 1972, for stimulating discussions.

I also wish to thank Professor Abdus Salam, the International Atomic Energy Agency and UNESCO for hospitality at the International Centre for Theoretical Physics, Trieste.

\section{Appendix A}

To make this paper notationally self-contained, we recall the following terminology. 
Definition A.1. A Feynman graph $G\left(V_{1} \ldots V_{m} ; \mathscr{L}\right)$ (with vertices $\left\{V_{1} \ldots V_{m}\right\}$ and a set of lines $\mathscr{L}=\mathscr{L}(G)$ ) is a graph associating to each line $l \in \mathscr{L}$ a propagator $\Delta_{l} \in \mathscr{S}^{\prime}\left(\mathbb{R}^{4}\right)$ whose Fourier transform has the form

$$
\tilde{\Delta}_{l}(p)=Z_{l}(p)\left(p^{2}-m_{l}^{2}+i 0\right)^{-1},
$$

$Z_{l}$ is a polynomial of degree $r_{l}$, and $m_{l}>0$ is the mass associated with the line. The superficial divergence of $G$ is

$$
\mu(G)=\sum_{l \in \mathscr{L}}\left(r_{l}+2\right)-4(m-1) .
$$

A subgraph $H \subset G$ is again a Feynman graph in an obvious way. We may take all vertices in $G$ to be external.

Definition A.2. The graph $G$ is one-particle irreducible (IPI), (onevertex irreducible (IVI)), if it is connected and cannot be disconnected by removal of one line (resp : vertex). Otherwise $G$ is called IPR (resp : IVR). A graph which is both IPI and IVI is said to be irreducible.

Definition A.3. A singularity family, (s-family), $\mathscr{E}$ for $G$ is a maximal collection of irreducible subgraphs of $G$ such that

a) If $H, H^{\prime} \in \mathscr{E}$, then $H \supset H^{\prime}, H^{\prime} \supset H$ or $\mathscr{L}(H) \cap \mathscr{L}\left(H^{\prime}\right)=\phi$ and

b) If $H_{1} \ldots H_{k} \in \mathscr{E}$ satisfy $\mathscr{L}\left(H_{i}\right) \cap \mathscr{L}\left(H_{j}\right)=\phi$ for any $i, j$ then $H=H_{1} \cup \cdots \cup H_{k}$ is not irreducible.

(Remark: An $\mathscr{E}$-family contains no "overlapping graphs".)

Definition A.4. A generalized vertex, $U$, is a non-empty subset $\left\{V_{1}^{\prime} \ldots V_{m}^{\prime}\right\}$ of the vertices of $G$. Given a generalized vertex $U$, the dominant subgraph $\hat{G}(U)$ of $G$ is formed by including all lines in $\mathscr{L}(G)$ joining any pair of vertices in $U$; if $H \subset G$ has vertices $U$, we write $\hat{H} \equiv G(U)$, the dominant graph for $H$ in $G$.

(Remark: In quantum electrodynamics, any irreducible graph is automatically dominant.)

For additive renormalization we require additional terminology. Given a Feynman graph $G$, the Feynman amplitude is the (formal) product of distributions

$$
\mathscr{T}_{G}=\prod_{l \in \mathscr{L}} \Delta_{l}\left(x_{l_{i}}-x_{l_{f}}\right),
$$

whose ill-definition necessitates renormalization. Suppose we have introduced a set of parameters $\omega$ in $\mathscr{T}_{G}$ such that $\mathscr{T}_{G}(\omega)=\Pi \Delta_{l}(\omega)\left(x_{l_{l}}-x_{l_{f}}\right)$ $\in \mathscr{S}^{\prime}\left(R^{4 m}\right)$ for a suitably large set $\omega$, depends meromorphically on $\omega$, and formally reduces to $\mathscr{T}_{G}$ as $\boldsymbol{\omega} \rightarrow \mathbf{0}$.

Definition A.5. Let $U=\left\{V_{1}^{\prime} \ldots V_{m}^{\prime}\right\}$ be a generalized vertex in $G$ and let $G^{\prime}=\hat{G}(U)$. A vertex part $\mathscr{X}$ for $U$ is a distribution (with support at 
$\left.x_{1}^{\prime}=\cdots=x_{m}^{\prime}\right)$ with the form

$$
\begin{aligned}
\tilde{X}(U ; \omega) & =1 \quad \text { if } m=1, \\
& =0 \quad \text { if } \quad G^{\prime} \text { is IPR, } \\
& =\delta\left(\sum_{i=1}^{m} p_{i}^{\prime}\right) P\left(\omega ; p_{1}^{\prime}, \ldots, p_{m}^{\prime}\right), \text { otherwise. }
\end{aligned}
$$

Here $P$ is a polynomial in $p^{\prime}$ of degree at most $\mu\left(G^{\prime}\right)$.

Definition A.6. A distribution $\mathscr{T} \in \mathscr{S}^{\prime}\left(\mathbb{R}^{4 m}\right)$ is an additively renormalized Feynman amplitude for $G\left(V_{1} \ldots V_{m}\right)$ if there exist a set of vertex parts $\mathscr{X}$ such that

$$
\mathscr{T}=\lim _{\omega \rightarrow 0} \sum_{P}\left(\prod_{l \in \mathscr{L}^{\prime}} \Delta_{l}(\omega)\left(x_{l_{i}}-x_{l_{f}}\right)\right)\left(\prod_{i=1}^{k(P)} \mathscr{X}\left(U_{i}^{P} ; \omega\right)\right)
$$

where the sum is taken over all partitions $P$ of $\left\{V_{1} \ldots V_{m}\right\}$ into $k(P)$ disjoint generalized vertices $U_{1}^{P} \ldots U_{k(P)}^{P}$.

Here

$$
\mathscr{L}^{\prime}=\mathscr{L}(G) \backslash \bigcup_{i=1}^{k(P)} \mathscr{L}\left(\hat{G}\left(U_{i}^{P}\right)\right)
$$

\section{Appendix B}

Proof of Theorem 3.1 (sketch). We draw heavily on the methods and results of Ref. [6] and refer the reader there for details.

Definition (Speer [6]):

For $K>0$, let $\Omega$ be the index set $\{1 \ldots K\}$. A family $\mathscr{E}$ of non-empty subset of $\Omega$ is called an s-family if

a) $\chi_{1}, \chi_{2} \in \mathscr{E} \Rightarrow \chi_{1} \subset \chi_{2}, \chi_{2} \subset \chi_{1}$ or $\chi_{1} \cap \chi_{2}=\phi$ and

b) for any $\chi \in \mathscr{E}, \chi \neq \bigcup_{\chi^{\prime} \in \mathscr{E}, \chi_{\ddagger}^{\prime} \chi} \chi^{\prime}$.

We let $\overline{\mathscr{E}}=\bigcup_{\chi \in \mathscr{E}} \chi ; \mathscr{E}$ is discrete if all elements of $\mathscr{E}$ are pairwise disjoint. For $\chi \in \mathscr{E}$, we let $\mathscr{E}(\chi)$ be the subfamily of $\mathscr{E}$ consisting of all sets which are proper subsets of $\chi$.

According to Ref. [6] Theorem 3.2, any analytic evaluator $\mathscr{V}$ may be decomposed relative to an analytic decomposition of the type given by Theorem 2.2 as

$$
\mathscr{V}_{\Omega}=\sum_{\chi \subset \Omega} \mathscr{S}(\chi)
$$


where $\mathscr{S}(\chi)$, the "singular part" associated with the variables in the set $\chi$, is defined on $f=\Sigma f(\cdot, \mathscr{E})$ by

$$
\mathscr{S}(\chi) f=\sum_{(\mathscr{E}, \mathscr{D})} \prod_{\psi \in \mathscr{D}}\left(\mathscr{V}_{\psi}-\mathscr{V}_{\overline{\mathscr{E}}(\psi)}\right) f(\cdot, \mathscr{E}) .
$$

The sum is over all $\mathscr{E}$ such that, for some discrete $\mathscr{D} \subset \mathscr{E}, \overline{\mathscr{D}}=\chi$. By convention, $\mathscr{S}(\phi)$ and $\mathscr{V}_{\phi}$ are the identity.

The applicability of these results is made possible by the remark that if $\mathscr{E}$ is an $s$-family for $G$, then the family $\{\mathscr{K}(H) ; H \in \mathscr{E}\}$ is an $s$-family for $\mathscr{K}(G)$ in the sense of the above definition. We may thus use the $d$-family $\mathscr{K}(G)$ as the index set $\Omega$.

We shall be content with exhibiting the vertex parts (i.e. counterterms) for a given analytic evaluator $\mathscr{V}$. The proof that $\mathscr{T}_{G}^{\prime}=\left(\mathscr{V}_{G}\right)(\mathbf{0})$ is an additive renormalization follows as in Ref. 6 by reorganization of terms, once we make the remark that in the case $\chi \subset \Omega=\mathscr{K}(G)$ contains an overlapping pair of graphs $H, H^{\prime}$, then

$$
\left(\mathscr{S}(\chi) \mathscr{T}_{G}\right)(\omega)=0
$$

(cf. Ref. [6], Remark 3.19a). ( $H$ and $H^{\prime}$ overlap if $\mathscr{L}(H) \cap \mathscr{L}\left(H^{\prime}\right) \neq \phi$ but neither $H \subset H^{\prime}$ nor $H^{\prime} \subset H$.)

For each $H \in \mathscr{K}(G)$, form subsets $\mathscr{N}(H) \subset \mathscr{K}(H)$ of nested sequences of graphs:

$$
\mathscr{N}(H)=\left\{H \supset H_{1} \supset \cdots \supset H_{n} ; H_{i} \in \mathscr{K}(H)\right\} ;
$$

we allow $\mathscr{N}(H)=\{H\}$. Set $\mathcal{N}^{\prime}(H)=\mathscr{N}(H) \backslash\{H\}$.

We claim that if $U=\left\{V_{1}^{\prime} \ldots V_{m}^{\prime}\right\}$ is a generalized vertex for $G$, and $G^{\prime}=G^{\prime}\left(V_{1}^{\prime} \ldots V_{m}^{\prime}\right)$, then $\mathscr{X}$ given by

$$
\mathscr{X}(U ; \omega)=\sum_{\mathscr{N}\left(G^{\prime}\right) \subset \mathscr{K}\left(G^{\prime}\right)} \mathscr{S}\left(\mathscr{N}\left(G^{\prime}\right)\right) \tau_{G^{\prime}}
$$

is a vertex part for $U$. The proof exactly parallels Ref. [6], Lemma 4.2.

Note that $\mathscr{X}=0$ if $G^{\prime}$ is IPR, in fact, if not irreducible. (The empty sum is 0 by convention.) This stronger condition on $\mathscr{X}$ still gives rise to an additive renormalization [11]. We remark, following Westwater [11], that once the vertex parts have been defined, we may set all $\omega$ equal to one complex parameter $\omega$. This makes contact with the method in Ref. [3].

Note also that by Ref. [6], Lemma 3.6, we may also write

$$
\mathscr{X}(U ; \omega)=\mathscr{S}\left(\left\{G^{\prime}\right\}\right) \sum_{\mathscr{N}^{\prime}\left(G^{\prime}\right) \mathcal{H}\left(G^{\prime}\right)} \mathscr{S}\left(\mathscr{N}^{\prime}\left(G^{\prime}\right)\right) \mathscr{T}_{G^{\prime}} .
$$

It may also be proved inductively that, for $G^{\prime}=G^{\prime}\left(V_{1}^{\prime} \ldots V_{m}^{\prime}\right)$ in the $d$-family $\mathscr{K}(G)$, the vertex part $\mathscr{X}\left(V_{1}^{\prime} \ldots V_{m}^{\prime}\right)$ satisfies the recursive relation

$$
\mathscr{X}\left(V_{1}^{\prime} \ldots V_{m}^{\prime} ; \omega\right)=\mathscr{S}\left(\left\{G^{\prime}\right\}\right) \sum_{P} \prod_{l \in \mathscr{L}^{\prime}} \Delta_{l}(\omega) \prod_{i=1}^{k(P)} \mathscr{X}\left(U_{i}^{P} ; \omega\right)
$$


where the sum runs over all partitions of $\left\{V_{1}^{\prime} \ldots V_{m}^{\prime}\right\}$ into disjoint generalized vertices $U_{1}^{P} \ldots U_{k}^{P}$. Here

$$
\mathscr{L}^{\prime}=\mathscr{L}\left(G^{\prime}\right) \backslash \bigcup_{i=1}^{k(P)} \mathscr{L}\left(\hat{G}\left(U_{i}^{P}\right)\right) .
$$

Note thus that (B.5) is reminiscent of the recursive definition in Ref. [7] of $\mathscr{X}$ with the operator $\mathscr{S}\left(\left\{G^{\prime}\right\}\right)$ playing the rôle of taking the Taylor series expansion of $\tilde{\mathscr{T}}_{G^{\prime}}$ up to order $\mu\left(G^{\prime}\right)$. Indeed it does [12].

\title{
References
}

1. Ashmore, J. F.: Lettere al Nuovo Cimento 4, 289 (1972).

2. Bollini, C. G., Giambiagi, J. J.: La Plata preprint, Febr. 1972.

3. t'Hooft, G., Veltman, M.: Utrecht preprint, Febr. 1972 (to be published in Nucl. Phys. B).

4. Speer,E.J.: J. Math. Phys. 9, 1404 (1968); Generalized Feynman Amplitudes. Princeton: University Press 1968.

5. Speer, E. J.: Commun. math. Phys. 23, 23 (1971).

6. See, however, Mitter, H., Breitenlohner, P.: Nucl. Phys. B7, 443 (1968).

7. Hepp, K.: Commun. math. Phys. 2, 301 (1966).

8. Appelquist, T.: Ann. Phys. (N.Y.) 54, 27 (1969).

9. Nakanishi, N.: Graph theory and Feynman integrals. New York: Gordon and Breach 1971

10. Lam, C.S.: Nuovo Cimento 59A, 397 (1969).

11. Westwater, M. J.: Fortschr. Phys. 17, 1 (1969).

12. In this connection see: Cicuta, G. M., Montaldi, E.: Lettere al Nuovo Cimento 4, 329 (1972).

\author{
J. F. Ashmore \\ School of Biological Sciences \\ University of Sussex \\ Falmer \\ Brighton, Sussex \\ Great Britain
}


